\title{
Linking the fractional derivative and the Lomnitz creep law to non-Newtonian time-varying viscosity
}

\author{
Vikash Pandey* and Sverre Holm \\ Department of Informatics, University of Oslo, P.O. Box 1080, NO-0316 Oslo, Norway
}

(Received 13 May 2016; published 23 September 2016; corrected 3 May 2018)

\begin{abstract}
Many of the most interesting complex media are non-Newtonian and exhibit time-dependent behavior of thixotropy and rheopecty. They may also have temporal responses described by power laws. The material behavior is represented by the relaxation modulus and the creep compliance. On the one hand, it is shown that in the special case of a Maxwell model characterized by a linearly time-varying viscosity, the medium's relaxation modulus is a power law which is similar to that of a fractional derivative element often called a springpot. On the other hand, the creep compliance of the time-varying Maxwell model is identified as Lomnitz's logarithmic creep law, making this possibly its first direct derivation. In this way both fractional derivatives and Lomnitz's creep law are linked to time-varying viscosity. A mechanism which yields fractional viscoelasticity and logarithmic creep behavior has therefore been found. Further, as a result of this linking, the curve-fitting parameters involved in the fractional viscoelastic modeling, and the Lomnitz law gain physical interpretation.
\end{abstract}

DOI: 10.1103/PhysRevE.94.032606

\section{INTRODUCTION}

Many complex media exhibit non-Newtonian behavior, meaning that stress no longer is proportional to strain rate. The behavior is mainly classified into two sub-categories: time independent and time dependent. The latter is still not so well understood despite its importance in applications. The underlying reason is the complexity of the phenomenon, and therefore models are often empirical and lack a proper physical interpretation [1].

When it comes to time-dependent non-Newtonian rheology, there exist two quite different approaches to modeling. On the one hand there are models based on time-varying viscosity giving rise to properties such as thixotropy and rheopecty. On the other hand, there have been advances in modeling time dependency with fractional calculus in which power laws are inherent [2-4]. These two approaches to modeling are quite distinct from each other and there are not many references from one field to the other. One of the intentions of this paper is to build a bridge between the two fields. The other is the derivation of Lomnitz's creep law.

The rest of the article is organized as follows. In the next two subsections we first give a short historical review of the power law responses and their modeling with fractional derivatives, which is then followed by a short summary of the difficulties associated with the modeling of time-varying viscous fluids. Subsequently a subsection is dedicated to the Lomnitz logarithmic creep law. Then in Sec. II we show how the fractional derivative naturally emerges from the relaxation modulus of a time-varying Maxwell model. The creep compliance of the model is identified as the Lomnitz law in Sec. III. Finally, in Sec. IV, we discuss the implications of this work.

\footnotetext{
*vikashp@ifi.uio.no
}

Published by the American Physical Society under the terms of the Creative Commons Attribution 3.0 License. Further distribution of this work must maintain attribution to the author(s) and the published article's title, journal citation, and DOI.

\section{A. Power law response}

The history of fractional derivatives in physics goes back to Abel [5], who in 1826 was the first to pose a mechanical problem, in the form of the isochrone curve, which required a fractional derivative of order $1 / 2$.

In the field of rheology, the paper [6] gives a good historical overview mentioning in particular two early contributions. The first, from 1921 [7], gave what is referred to as Nutting's equation. It is the power law stress response of a fluid to a constant strain load:

$$
\sigma(t) \propto t^{-\alpha}, \quad 0<\alpha<1 .
$$

It was found to describe many media better than the usual decaying exponential response. The next significant contribution is Scott Blair's fractional model based on the rheology of, e.g., cheese and clay [8]. He found that the rheological law underlying the Nutting equation was actually a fractional order differential equation, where the fractional derivative of order $\alpha$ is a convolution between a first order derivative and a power law kernel:

$$
\frac{\partial^{\alpha}}{\partial t^{\alpha}} f(t)=\dot{f}(t) * \frac{t^{-\alpha}}{\Gamma(1-\alpha)}, \quad 0<\alpha<1,
$$

where $\Gamma(\cdot)$ is the gamma function.

Since the time of Nutting and Scott Blair, the fractional model in rheology has been much developed as a property describing a time-dependent non-Newtonian fluid with properties interpolating between a Hookean and a Newtonian one. This has resulted in the generalization of the classical Kelvin-Voigt, Maxwell, and Zener spring-dashpot models into corresponding fractional ones. Likewise, the mathematical theory of fractional differential equations has been developed [9].

A recent textbook covering fractional viscoelasticity is [3]. However, despite the vast development of fractional derivatives as an attractive tool of engineering analysis over the last decades, the use of fractional derivatives in viscoelasticity is essentially motivated by the same power law creep as at the time of Nutting and Scott Blair. Even at present there is no physical interpretation of the important parameter of the fractional order [10]. This has apparently reduced the 
credibility of fractional viscoelastic models and so restrained them to the realm of empirical "curve fits," though their fit to real data in, e.g., biology [11] and porous media [12] has been established [13].

The main reason is that little work was done that demonstrated a deductive approach to the fractional derivatives. One of the main contributions came from Bagley and Torvik as they established a direct connection between fractional derivatives and physical processes underlying polymer dynamics. They studied the Rouse model, which does not include hydrodynamic and volume interactions; however, it has been the basis of molecular theories for the dynamics of polymer solids with no crosslinking $[14,15]$. It was shown that fractional forcedisplacement relationships emerging from the microscopic dynamics of the polymer constituents translate into a fractional stress-strain relationship of order $1 / 2$, which also dictates the macroscopic mechanical properties. Besides being justified by the physical principles governing the polymer behavior, the thermodynamic consistency of the fractional models is also ensured [16]. Recently the possibility to extend the order of the fractional derivative to 0 and 1 was also illustrated by generalizing the Rouse relaxation time [17]. An alternative approach which ensures the realizability of fractional viscoelastic models is through hierarchical arrangements of springs and dashpots in the form of trees, ladders, and fractal networks [18]. This implies that fractional derivatives naturally arise when constitutive properties of a large number of classical viscoelastic elements are combined.

Possibly the first suggestion to use fractional derivatives came from Gemant [19] in order to model wave properties which varied as frequency raised to fractional powers [20]. This can be linked to the property that the Fourier transform of the fractional derivative is a power law,

$$
\mathcal{F}\left(\frac{d^{\alpha}}{d t^{\alpha}} f(t)\right)=(i \omega)^{\alpha} F(\omega) .
$$

The fact that Eq. (3) also can be seen as a direct extension from the regular integer-order derivative motivates its use [21,22].

Even in that field, the status is that it lacks a proper physical interpretation. It should also be noted that the fractional viscoelasticity model is an alternative to power law attenuation caused by the different physical model of multiple scattering in fractal media [23].

\section{B. Time-varying viscosity}

Another way to describe a time-dependent non-Newtonian fluid is with a time-varying viscosity. When it increases with time it results in a shear-thickening property called rheopecty. It is less common than the opposite shear-thinning property, thixotropy. Some examples of thixotropic fluids are paint, honey, coal-water slurries, and waxy crude oil. The bovine synovial fluid found in moving joints and the cytoplasm of cells are rheopectic and thixotropic respectively. Examples of rheopecty are a particular suspension involving clay, calcium carbonate, starch, and water [24], as well as cream; the longer one whips, the thicker it gets.

We follow [25] in distinguishing between time-dependent and time-independent non-Newtonian fluids. Rheopecty and thixotropy should therefore not be confused with time- independent behavior where viscosity varies only with the strain rate [26]. One way to describe that is with a power law in the strain derivative, as in the Herschel-Bulkley model $\sigma(t)=\sigma_{t}+m(\dot{\varepsilon})^{n}$, where $\sigma$ and $\varepsilon$ are the shearing stress and strain respectively.

The model has a threshold, $\sigma_{t}$, below which the fluid does not flow. Dilatant behavior occurs if $n>1$ and pseudoplastic properties are described with $n<1$. If $n=1$ this is the Bingham model and if the threshold is also removed, i.e., $\sigma_{t}=0$, it describes Newtonian viscous behavior [27]. The constant $m$ is the consistency index.

The Herschel-Bulkley model and more complex variants of it may describe time-dependent viscosity if the consistency index is a function of time, $m(t)$, combined with $n=1$ in order to avoid a mix between time and strain rate dependency [25]. One recent development is to introduce time dependency by replacing the second term of the Herschel-Bulkley model with a fractional derivative [27]. The motivation is, however, only phenomenological. Further, it must be noted that an arbitrary substitution has its drawbacks, as sometimes it could lead to unphysical results.

Nevertheless, the status is that the behavior of thixotropic fluids is far from being understood [28] and that present models cannot predict all the properties of concentrated suspensions [1]. Interestingly, [29] states that the model for, e.g., laterite slurries lacks a way of predicting the relaxation time as it depends on the shear history.

Here we will show that time-dependent non-Newtonian properties are more closely linked to fractional viscoelasticity than previously thought. That is done by first assuming a time-varying viscosity, $\eta(t)$,

$$
\sigma_{d}(t)=\eta(t) \dot{\varepsilon}_{d}(t), \quad \eta(t)>0,
$$

and then combining that with a spring in series, yielding a time-varying Maxwell model. It is then shown that in the special case of a linearly time-varying viscosity, the fluid's response is a power law as in the Nutting equation. In this way a link is found between the two disparate fields of fractional viscoelasticity and time-dependent non-Newtonian rheology. An interpretation of the fractional order and the relaxation time then follows from this bridging.

\section{Lomnitz's creep law}

In 1956 Lomnitz proposed a logarithmic creep law [30] to describe the creep behavior of igneous rocks:

$$
\varepsilon(t)=\frac{\sigma}{E_{0}}[1+q \ln (1+a t)], \quad t \geqslant 0,
$$

where $E_{0}$ is the shear modulus. The parameter $a$ is a positive material constant, and to ensure the dimensional consistency of the equation it must relate to the relaxation time during which the transition from the elastic- to creep-type deformation occurs. The beauty of the Lomnitz law lies in the fact that as $t \rightarrow 0$ there is no singularity observed in it. To the best of our knowledge, a derivation of the creep law has never been obtained. Having introduced it as an empirical law, the dimensionless creep constant $q$ is estimated from curve fitting, from which very low values of $q \ll 1$ are noticed [30]. Further, the coefficient $a$ must be chosen large enough such 
that $a t \gg 1$. The creep law has been successfully employed in mantle rheology, so stress wave attenuation in the earth could be modeled [31].

The logarithmic term in Eq. (5), when generalized by Jeffreys in 1958 [32], was shown to interpolate creep data between a logarithmic and a linear law which ensures its suitability for wide applications in seismology. An interesting application of the law was demonstrated in explaining the Chandler wobble, the nutation of Earth's rotational axis. The suitability of the Lomnitz law and the Jeffreys-Lomnitz law to curve-fit the time-dependent earthquake creep data can be found in [33]. The generalized law was further extended by Strick who also established the link between the extended Jeffreys-Lomnitz creep law and the tapered (nonuniform) ladder network of springs and dashpots [34] which is similar to the realizability of fractional derivatives, as mentioned earlier. This observation hints about a common underlying physical mechanism behind the fractional power-law behavior and logarithmic creep law behavior, which we will identify as the time-varying non-Newtonian viscosity in the next two sections.

\section{TIME-VARYING VISCOSITY AS A SPRINGPOT}

Independent of both the fields of fractional viscoelasticity and non-Newtonian rheology, in [35] it was found that the friction between grains in a fluid-saturated sediment may be described by a time-varying Maxwell model, which consists of a spring in series with the viscosity given in Eq. (4). The link to non-Newtonian media was not so apparent, since rather than being called a rheopectic model it was described as a strainhardening one. That terminology is used in, e.g., seismics, but more often strain hardening describes a non-Hookean medium where the elastic modulus varies with applied stress.

In order to deal with the time-varying viscosity of Eq. (4), the viscosity of the damper was approximated to vary linearly with time in [35]:

$$
\sigma_{d}(t)=\left[\eta_{0}+\theta t\right] \dot{\varepsilon}_{d}(t), \quad t \geqslant 0 .
$$

The simplest way to ensure a positive viscosity is to deal only with rheopectic materials with $\theta>0$.

In the modified Maxwell model, as illustrated in Fig. 1(a), the stresses in the elements in the series combination are the same, i.e., $\sigma(t)=\sigma_{s}(t)=\sigma_{d}(t)$, where the subscripts denote the contribution from the spring and the dashpot respectively. The strains will add and so do their derivatives:

$$
\dot{\varepsilon}=\dot{\varepsilon}_{s}+\dot{\varepsilon}_{d} .
$$

Substituting the response of the spring, $\sigma_{s}=E_{0} \varepsilon_{s}$, and the dashpot, Eq. (6), gives

$$
\frac{\dot{\sigma}}{E_{0}}+\frac{\sigma}{\eta_{0}+\theta t}=0
$$

In deriving this result, we have set the strain input to a unit step. The resulting stress response, also called the relaxation modulus, is derived in Appendix 1 as

$$
G(t, 0)=\left(1+\frac{\theta}{\eta_{0}} t\right)^{-E_{0} / \theta}, \quad \theta>0, \quad t \geqslant 0 .
$$

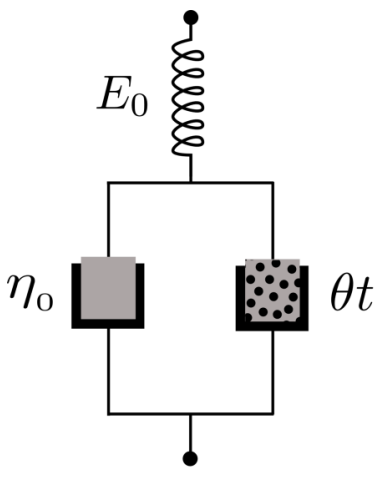

(a)

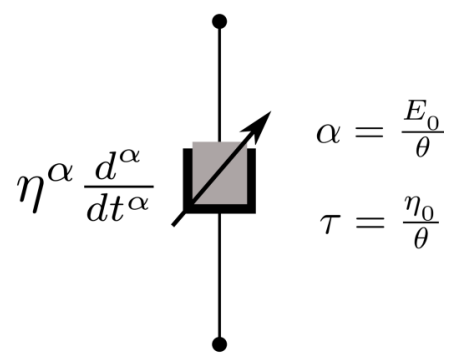

(b)
FIG. 1. (a) Maxwell spring damper with time-varying viscosity. (b) The fractional dashpot with similar relaxation modulus

It has two temporal arguments since it is the response of a time-varying system. The second argument is the time of the input relative to the start of the onset of the time variation of the viscosity of Eq. (6). This is the key difference between linear viscoelasticity, and the time-dependent non-Newtonian fluid properties of thixotropy and rheopecty. The former is a time-invariant property where the structural elements of the medium remain unchanged in time; the latter, in contrast, is a time-variant property where the structural units could either build up or break down with respect to time. If the constant dashpot is small compared to the time-varying part, the exact step response can be approximated as

$$
\begin{aligned}
G(t, 0) & =G_{F D}(t+\tau) \approx G_{F D}(t)=\left(\frac{t}{\tau}\right)^{-\alpha}, \\
\tau & =\eta_{0} / \theta, \quad \alpha=E_{0} / \theta, \quad \eta_{0} \ll \theta t,
\end{aligned}
$$

where $G_{F D}(t)$ is the relaxation modulus of the fractional dashpot but without the scaling parameter of $1 / \Gamma(1-\alpha)$. This result was derived in [35], but the significance of the result was not noted because there was no mention of either the connection to past work on power law responses or to fractional derivatives. Here we observe that the similarity with Eq. (1) establishes the similarity between the Nutting equation and the linearly time-varying viscosity.

It is also worthwhile to note that the exponent $\alpha$ is a measure of the interplay between elasticity and viscosity. That agrees with the intuitive observation of [36] regarding "firmness" of the material where they referred to $\alpha$ as the coefficient of dissipation. That link can be further elaborated by noting the following Fourier relationship (Ref. [9], p. 110):

$$
f(t)=\frac{t^{-\alpha}}{\Gamma(1-\alpha)}, t>0 \quad \Leftrightarrow \quad F(\omega)=(i \omega)^{\alpha-1} .
$$

Thus the step response has a power law Fourier transform $G(\omega)=\Gamma(1-\alpha) \tau^{\alpha}(i \omega)^{\alpha-1}$, meaning that the transform of the impulse response, $h(t)$, is $H(\omega)=i \omega G(\omega)=\Gamma(1-$ $\alpha) \tau^{\alpha}(i \omega)^{\alpha}$. As already noted, this is also the Fourier transform of a fractional derivative, Eq. (3). Therefore the stress is given as a convolution with a fractional derivative operator:

$$
\sigma(t)=h(t) * \varepsilon(t), \quad h(t)=\frac{1}{\Gamma(1-\alpha) \tau^{\alpha}} \frac{d^{\alpha}}{d t^{\alpha}}(\cdot) .
$$


The fractional derivative is the response of a fractional dashpot of order $\alpha$, also called a springpot or a Scott Blair element [3], as shown in Fig. 1(b). This description is similar to the linearly time-varying viscosity of Eq. (6) in series with a spring as shown in Fig. 1(a), and this is our first finding. In this way a link has been found between the disparate fields of time-varying viscosity and fractional viscoelastic materials.

By analogy, the same procedure can be used to find the equivalent of a fractional capacitor [37] by replacing the spring with a resistor, and the dampers by capacitors. A time-varying capacitor could be due to a time-varying dielectric.

\section{TIME-VARYING VISCOSITY AS LOMNITZ'S CREEP LAW}

The creep compliance of the time-varying Maxwell model is its strain response to a unit step in stress, and can be found in a similar way as the relaxation modulus; see Appendix 2:

$$
J(t, 0)=1+\alpha \ln \left(1+\frac{t}{\tau}\right) .
$$

Surprisingly Eq. (13) is actually the time varying part of Lomnitz's creep law expressed by Eq. (5). Comparing the two equations and then relating them to Eq. (10), we identify the creep constant, $q=\alpha=E_{0} / \theta$, and the coefficient, $a=$ $1 / \tau=\theta / \eta_{0}$. Thus we obtain a derivation of the logarithmic creep law, and also extract physical interpretation of the terms involved. This is our second finding.

The creep compliance of the time-varying Maxwell model is therefore rather different from that of the fractional dashpot, $J_{F D}(t)=1 / \Gamma(1+\alpha)(t / \tau)^{\alpha}$. For the fractional dashpot, $J_{F D}(t)$ must remain finite and therefore the order, $\alpha$, cannot exceed 1 [3]. However, a similar criterion could not be found directly for the time-varying Maxwell model. It should also be noted that the reciprocity principle of [3], which links the Laplace transforms of the creep compliance and the relaxation modulus, is not satisfied here. This is because the time-varying Maxwell model is not a time-invariant linear system, as assumed by the application of the Laplace transforms.

\section{CONCLUSION}

In this work we have achieved two goals. First, it has been shown that a linearly time-varying non-Newtonian viscosity has the same power-law relaxation modulus as the linear viscoelasticity described by the fractional derivative element called a springpot. This means that the rather disparate fields of fractional viscoelasticity and time-dependent non-Newtonian rheology are more closely linked than previously thought. It has also been shown that the order of a fractional element is the ratio of the elastic part and the coefficient of the time-varying viscosity. This opens up for a more physical interpretation of the fractional order. Fractional derivative stress-strain constitutive relationships not only adequately describe the mechanical properties of complex materials, their Fourier transform utilities also lead to closed-form solutions while dealing with wave dispersive properties of materials. The correct knowledge of the fractional order could then limit the ambiguities in the curve fitting of dispersion plots. This also gives an interpretation of fractional viscoelasticity in terms of rheopecty. Most examples in rheopecty are for fluids with very slowly varying viscosities, in the order of seconds. The result derived here could indicate that these properties exist at much shorter time scales as well and thus may influence elastic wave propagation in the $\mathrm{kHz}$ and $\mathrm{MHz}$ ranges also.

Second, we showed that the creep compliance of the linearly time-varying non-Newtonian viscosity is actually Lomnitz's creep law. This may possibly be the first such deductive derivation of the Lomnitz law. Moreover, similar to the case of fractional derivatives, the parameters of the creep law used for curve fitting have a physical interpretation now. The coefficient $a=\theta / \eta_{0}$ from Eq. (13), when seen in light of the constraint $a t \gg 1$ imposed by Lomnitz [30], leads to $\eta_{0} \ll \theta t$. As is evident, the assumption $\eta_{0} \ll \theta t$ is common to both Nutting's law and Lomnitz's law, and thus indicates a common underlying physical mechanism. The mechanism is essentially a non-Newtonian time-varying viscosity of which the varying part increases linearly with time and dominates over the constant part. Further, Lomnitz's law has been found to be satisfied both at short and large time scales for igneous rocks. This implies that, in order for $\eta_{0}$ to be much smaller than $\theta t$, the time-varying part of the viscosity $\theta$ must have a very large value. Furthermore, the observation regarding the small values of the creep constant $q \ll 1$ obtained for igneous rocks [30], when analyzed using our finding $q=E_{0} / \theta$, implies that the time-varying part of the viscosity dominates over the elasticity of the rocks, i.e., $\theta \gg E_{0}$.

This contribution also outlines a way in which linear acoustics may develop from its roots in the Hookean and Newtonian assumptions. Non-Hookean behavior is the main source of nonlinearity in addition to convection in the mature field of nonlinear acoustics [38]. A much less developed area of study is that of time-dependent non-Newtonian behavior. It may be one of the main sources of power law characteristics of complex media. It follows that it may also be a major cause of power law behavior in both the dispersion and attenuation of propagating waves.

Finally, we summarize that fractional derivatives and Lomnitz law arise naturally from time-varying viscoelasticity. Though the derivation is from a viscoelastic model only, we ultimately aim to discover the multifacets of fractional calculus. The overall goal is to change the "empirical only" attitude which has been towards fractional viscoelastic models and Lomnitz's law. Further, the fractional viscoelastic models are not contrary to the traditional viscoelastic models, but rather they should be viewed as an extension of the latter. We expect that the findings of this article will increase the degree of confidence in employing fractional derivatives and Lomnitz's creep law in the modeling of complex material behavior which is encountered in rheological studies of colloids, food and pharmaceutical products, and biological and earth materials.

\section{ACKNOWLEDGMENTS}

We thank the reviewers for good questions that eventually led to the discovery of the relationship with the Lomnitz law. This project has received funding from the European Union's Horizon 2020 research and innovation programme under Grant Agreement No. 668039. This paper reflects only the authors' 
view. The European Commission is not responsible for any use that may be made of the information it contains.

\section{APPENDIX: THE RESPONSE OF A TIME-VARYING DASHPOT IN SERIES WITH A SPRING}

\section{The relaxation modulus}

Integrating on both sides of Eq. (8) for $\theta>0$ yields

$$
\frac{1}{E_{0}} \ln \sigma=-\left\{\frac{1}{\theta} \ln \left(\eta_{0}+\theta t\right)+\ln C\right\},
$$

where $\ln C$ is a constant of integration. Thus

$$
\ln \sigma=-\left\{\frac{E_{0}}{\theta} \ln \left(\eta_{0}+\theta t\right)+E_{0} \ln C\right\} .
$$

Imposing the initial condition that at $t=0$ the dashpot has not had time to react, and so all the strain is taken by the spring, gives $\sigma(t=0)=\sigma_{0}=E_{0} \varepsilon_{0}$. Using this result in Eq. (A2) gives

$$
\ln \sigma_{0}=-\left\{\ln \eta_{0}^{\frac{E_{0}}{\theta}}+\ln C^{E_{0}}\right\}=\ln \left(C \eta_{0}^{\frac{1}{\theta}}\right)^{-E_{0}},
$$

and the constant is

$$
C^{-E_{0}}=\frac{\sigma_{0}}{\eta_{0}^{\frac{-E_{0}}{\theta}}}
$$

Rewriting Eq. (A2) gives

$$
\ln \sigma=\ln \left\{C\left(\eta_{0}+\theta t\right)^{\frac{1}{\theta}}\right\}^{-E_{0}}
$$

or

$$
\sigma=\left\{C\left(\eta_{0}+\theta t\right)^{\frac{1}{\theta}}\right\}^{-E_{0}}=\sigma_{0}\left(1+\frac{\theta}{\eta_{0}} t\right)^{-\frac{E_{0}}{\theta}} .
$$

The desired unit step response of Eq. (9) is found by setting $\sigma_{0}=1$.

\section{The creep response}

The input for the creep response is a step in stress, the opposite of the case for finding the relaxation modulus. The stress and strain then follow:

$$
\frac{\sigma}{\eta_{0}+\theta t}=\dot{\varepsilon}, \quad t>0
$$

Since the total stress in the two dashpots in parallel combination is the same as the stress in the spring in series, Eq. (A7) becomes

$$
\frac{E_{0} \varepsilon_{s}}{\eta+\theta t}=\dot{\varepsilon}
$$

Now, following the same steps as in the calculation of relaxation modulus, integration of Eq. (A8) gives

$$
\varepsilon=\frac{E_{0} \varepsilon_{s}}{\theta} \ln \left(\eta_{0}+\theta t\right)+\ln C,
$$

where, $\ln C$ is a constant of integration. On imposing the initial condition at $t=0$, total strain is limited to the strain in the spring, $\varepsilon=\varepsilon_{s}$, we get

$$
\ln C=\left(1-\frac{E_{0}}{\theta} \ln \eta_{0}\right) \varepsilon_{s} .
$$

Substituting Eq. (A10) in Eq. (A9), and setting $\varepsilon_{s}=1$, we obtain the creep compliance of Eq. (13) as

$$
\begin{aligned}
\varepsilon & =1+\frac{E_{0}}{\theta}\left\{\ln \left(\eta_{0}+\theta t\right)-\ln \eta_{0}\right\} \\
& =1+\frac{E_{0}}{\theta} \ln \left(1+\frac{\theta}{\eta_{0}} t\right) .
\end{aligned}
$$

[1] P. Coussot and C. Ancey, Phys. Rev. E 59, 4445 (1999).

[2] M. M. Meerschaert, D. A. Benson, H.-P. Scheffler, and B. Baeumer, Phys. Rev. E 65, 041103 (2002).

[3] F. Mainardi, Fractional Calculus and Waves in Linear Viscoelesticity: An Introduction to Mathematical Models (Imperial College Press, London, 2010), pp. 1-347.

[4] R. Garra, Phys. Rev. E 84, 036605 (2011).

[5] N. H. Abel, J. Reine. Angew. Math. 1, 153 (1826).

[6] S. Rogosin and F. Mainardi, (2014), doi:10.1685/journal.caim.481.

[7] P. Nutting, J. Franklin. Inst. 191, 679 (1921).

[8] G. W. Scott Blair and M. Reiner, Appl. Sci. Res. 2, 225 (1951).

[9] I. Podlubny, Fractional Differential Equations (Academic Press, New York, 1999).

[10] J. A. T. Machado, F. Mainardi, and V. Kiryakova, Fract. Calc. Appl. Anal. 18, 495 (2015).

[11] F. Meral, T. Royston, and R. Magin, Commun. Nonlinear Sci. 15, 939 (2010)

[12] J. M. Carcione, F. J. Sanchez-Sesma, F. Luzón, and J. J. P. Gavilán, J. Phys. A: Math. Theor. 46, 345501 (2013).

[13] S. P. Näsholm and S. Holm, Fract. Calc. Appl. Analysis 16, 26 (2013).
[14] P. E. Rouse, J. Chem. Phys. 21, 1272 (1953).

[15] R. L. Bagley and P. J. Torvik, J. Rheol. 27, 201 (1983).

[16] R. L. Bagley and P. J. Torvik, J. Rheol. 30, 133 (1986).

[17] A. W. Wharmby and R. L. Bagley, J. Rheol. 57, 1429 (2013).

[18] H. Schiessel, R. Metzler, A. Blumen, and T. F. Nonnenmacher, J. Phys. A: Math. Gen. 28, 6567 (1995).

[19] A. Gemant, J. Appl. Phys. 7, 311 (1936).

[20] T. L. Szabo and J. Wu, J. Acoust. Soc. Am. 107, 2437 (2000).

[21] W. Chen and S. Holm, J. Acoust. Soc. Am. 114, 2570 (2003).

[22] S. Holm and R. Sinkus, J. Acoust. Soc. Am. 127, 542 (2010).

[23] S. A. Lambert, S. P. Näsholm, D. Nordsletten, C. Michler, L. Juge, J.-M. Serfaty, L. Bilston, B. Guzina, S. Holm, and R. Sinkus, Phys. Rev. Lett. 115, 094301 (2015).

[24] J. Yannas and R. Gonzalez, Nature (London) 191, 1384 (1961).

[25] R. P. Chhabra, Rheology of Complex Fluids (Springer, New York, 2010), pp. 3-34.

[26] J. R. Royer, D. L. Blair, and S. D. Hudson, Phys. Rev. Lett. 116, 188301 (2016).

[27] D. Yin, W. Zhang, C. Cheng, and Y. Li, J. Non-Newtonian Fluid Mech. 187-188, 32 (2012).

[28] P. R. de Souza Mendes, J. Non-Newtonian Fluid Mech. 164, 66 (2009). 
[29] B. C. Blakey and D. F. James, Int. J. Miner. Process. 70, 23 (2003).

[30] C. Lomnitz, J. Geol. 64, 473 (1956).

[31] C. Lomnitz, J. Geophys. Res. 67, 365 (1962).

[32] H. Jeffreys, Geophys. J. R. Astron. Soc. 1, 92 (1958).

[33] D. J. Darby, New Zeal. J. Geol. Geop. 32, 129 (1989).

[34] E. Strick, J. Geophys. Res. 89, 437 (1984).
[35] M. J. Buckingham, J. Acoust. Soc. Am. 108, 2796 (2000).

[36] G. W. Scott Blair and F. Coppen, Am. J. Psychol. 55, 215 (1942).

[37] S. Westerlund and L. Ekstam, IEEE Trans. Dielectr. Electr. Insul. 1, 826 (1994).

[38] M. F. Hamilton and D. T. Blackstock, Nonlinear Acoustics (Academic, New York, 1998). 